\title{
Contraste de la distribución Logística Generalizada en 31 registros históricos de eventos máximos anuales
}

\author{
Contrast of Generalized Logistic Distribution in 31 Historical Records \\ of Annual Extreme Events
}

\author{
Campos-Aranda D.F. \\ Facultad de Ingeniería \\ Universidad Autónoma de San Luis Potosí \\ Correo:campos_aranda@hotmail.com
}

Información del artículo: recibido: noviembre de 2011, aceptado: junio de 2012

\begin{abstract}
Resumen
La distribución Logística Generalizada es la más reciente cuya aplicación ha sido establecida bajo precepto, por ello es importante su contraste con las otras dos que le precedieron, la Log-Pearson tipo III en USA y la General de Valores Extremos en Inglaterra. Se comenzó por destacar la importancia en la estimación de las crecientes de diseño, de los análisis probabilísticos y de las distribuciones citadas. Para la más reciente se describe con detalle la estimación de sus tres parámetros de ajuste por el método de momentos L. Además se propone su ajuste por minimización del error cuadrático medio a través optimización numérica. Los resultados de la aplicación de esta distribución en 31 registros de eventos máximos anuales, con base en los dos métodos citados, se contrastan con los óptimos obtenidos previamente con los modelos General de Valores Extremos y Log-Pearson tipo III. Se concluye que la distribución Logística Generalizada (LOG) es una opción conveniente para registros que muestran grandes cocientes $L$ de curtosis y que en general sus resultados conducen a las predicciones más severas en los periodos de retorno extremos, en registros con valores dispersos.
\end{abstract}

\section{Descriptores:}

- distribución LOG

- momentos $L$

- cocientes de momentos $L$

- diagrama de cocientes de momentos $L$

- optimización numérica 


\begin{abstract}
The Generalized Logistic distribution is the most recent whose application has been established as precept. Thus, comparing it with the other two that preceded it: the Log-Pearson type III in USA and the General Extreme Values in England, is of high importance. In this work, the relevance of probabilistic analysis and the above mentioned distributions in design flood estimation is pointed out. For the most recent, a fitting method of L-moments is described in detail for the estimation of its three parameters, also a fitting by minimizing the quadratic mean error through numerical optimization is proposed. The results of the application of this distribution to 31 records, using both cited methods, are compared with the optimal ones obtained using the General Extreme Values and Log-Pearson type III models. It is concluded that the Generalized Logistic distribution is a good choice for records with high L-kurtosis quotients and its predictions in general are more extreme in high return periods when applied to records with outliers.
\end{abstract}

\section{Introducción}

Las estimaciones fundamentales de los hidrólogos están relacionadas con el escurrimiento en dos escalas de tiempo, la mensual y la instantánea. En el primer caso, se buscan los volúmenes escurridos disponibles para el diseño hidrológico de embalses de aprovechamiento. En el segundo caso, se intenta obtener las crecientes que generan la cuenca estudiada, asociadas éstas a diversas probabilidades de excedencia, cuyo recíproco es el periodo de retorno o intervalo promedio de recurrencia en años. Las crecientes son básicas en el diseño y la revisión hidrológica de todo tipo de obras hidráulicas de protección como son: presas, diques, rectificaciones y encauzamientos; además permiten el dimensionamiento de las obras de cruce (alcantarillas y puentes).

La estimación de crecientes de diseño más confiable es la que está basada en los registros de gastos máximos anuales, existiendo actualmente dos enfoques de procesamiento, el local y el regional. El tratamiento local de los datos disponibles se aplica donde el registro es amplio (> 50 años), si es menor, pero cuenta con más de 25 años, los resultados de procesamiento local se deben ratificar mediante comparación con cuencas vecinas (WRC, 1977). Cuando el registro tiene menos de 25 años las estimaciones de crecientes deben estar basadas en el enfoque regional.

La estimación probabilística de crecientes ha evolucionado desde sus inicios en 1930 y 1941 con los estudios de Hazen y Gumbel (Maidment, 1993), hasta el uso de modelos probabilísticos físicamente basados. Una etapa importante aconteció a mediados de los años sesenta, cuando el Subcomité de Hidrología del US Water
Resources Council, contrastó seis distribuciones comúnmente empleadas en esa época y concluyó que el modelo Log-Pearson tipo III (LP3) era el más conveniente y lo recomendó para uso general en las agencias de gobierno (Ponce, 1989). En un estudio similar en Inglaterra se determinó que las distribuciones de tres parámetros de ajuste (General de Valores Extremos, Pearson tipo III y LP3) conducían a mejores ajustes que los modelos de dos parámetros (NERC, 1975).

La propuesta de uso generalizado de la distribución general de valores extremos (GVE) incluye como caso especial a la distribución Gumbel, la cual ya era conocida y utilizada; además define a los modelos Log-Gumbel y Weibull, que son curvas en el papel de probabilidad Gumbel-Powell, el primero con concavidad hacia arriba y el segundo hacia abajo. La nueva versión del Flood Studies Report (NERC, 1975) se llama Flood Estimation Handbook y recomienda para los análisis de frecuencia de crecientes a la distribución Logística Generalizada, ajustada mediante el método de los momentos $L$, que es más confiable y consistente en registros sesgados (Mansell, 2003; Shaw et al., 2011).

Ya se han realizado contrastes de las distribuciones GVE y LP3 (Campos, 2001 y 2002a, b) en 31 registros históricos de eventos máximos anuales con amplitudes variando de 16 a 113 años. Por tanto, el objetivo de este estudio fue aplicar la distribución Logística Generalizada (LOG) a tales registros históricos mediante dos métodos de ajuste, el de momentos $L$ y el de optimización numérica y confrontar sus resultados con los óptimos obtenidos para los modelos citados, finalmente formular conclusiones relativas a esta última distribución. 


\section{Desarrollo}

Momentos y cocientes $L$ muestrales

Son un sistema alternativo para describir las formas de las funciones de distribución de probabilidades (FDP). Históricamente aparecen como modificaciones de los momentos de probabilidad pesada (MPP) desarrollados por Greenwood et al. (1979). Los momentos L son combinaciones lineales de los $M P P$, de manera que (Hosking y Wallis, 1997):

$\lambda_{1}=\beta_{0}$

$\lambda_{2}=2 \cdot \beta_{1}-\beta_{0}$

$\lambda_{3}=6 \cdot \beta_{2}-6 \cdot \beta_{1}+\beta_{0}$

$\lambda_{4}=20 \cdot \beta_{3}-30 \cdot \beta_{2}+12 \cdot \beta_{1}-\beta_{0}$

Además se definen los cocientes $(\tau)$ de momentos $L$, comenzando con $L-C v$ que es análogo al coeficiente de variación y después los de similitud con los coeficientes de asimetría (Cs) y de curtosis $(C k)$ :

$\tau_{2}=\lambda_{2} / \lambda_{1}$

$\tau_{3}=\lambda_{3} / \lambda_{2}$

$\tau_{4}=\lambda_{4} / \lambda_{2}$
En una muestra de tamaño $n$, con sus elementos arreglados en orden ascendente $\left(x_{1} \leq x_{2} \leq \cdots \leq x_{n}\right)$ los estimadores insesgados de $\beta_{r}$ son:

$b_{0}=\frac{1}{n} \sum_{j=1}^{n} x_{j}$

$b_{1}=\frac{1}{n} \sum_{j=2}^{n} \frac{(j-1)}{(n-1)} x_{j}$

$b_{2}=\frac{1}{n} \sum_{j=3}^{n} \frac{(j-1) \cdot(j-2)}{(n-1) \cdot(n-2)} x_{j}$

$b_{3}=\frac{1}{n} \sum_{j=4}^{n} \frac{(j-1) \cdot(j-2) \cdot(j-3)}{(n-1) \cdot(n-2) \cdot(n-3)} x_{j}$

Los estimadores muestrales de $\lambda_{r}$ serán $l_{r}$ estando definidos por las ecuaciones 1 a 4 y los de los cocientes serán $t_{2}, t_{3}$ y $t_{4}$, según las ecuaciones 5 a 7 .

\section{Diagrama de momentos $L$}

Hosking y Wallis (1997) establecieron la relación que guardan los cocientes de momentos $L$ de asimetría y curtosis en cinco distribuciones de probabilidad (figura 1): logística generalizada (LOG), general de valores extremos (GVE), Log-Normal de 3 parámetros (LN3), Pearson tipo III (PT3) y pareto generalizada (PAG).
Figura 1. Ubicación de los 31 registros históricos procesados en el diagrama de cocientes de momentos $L$

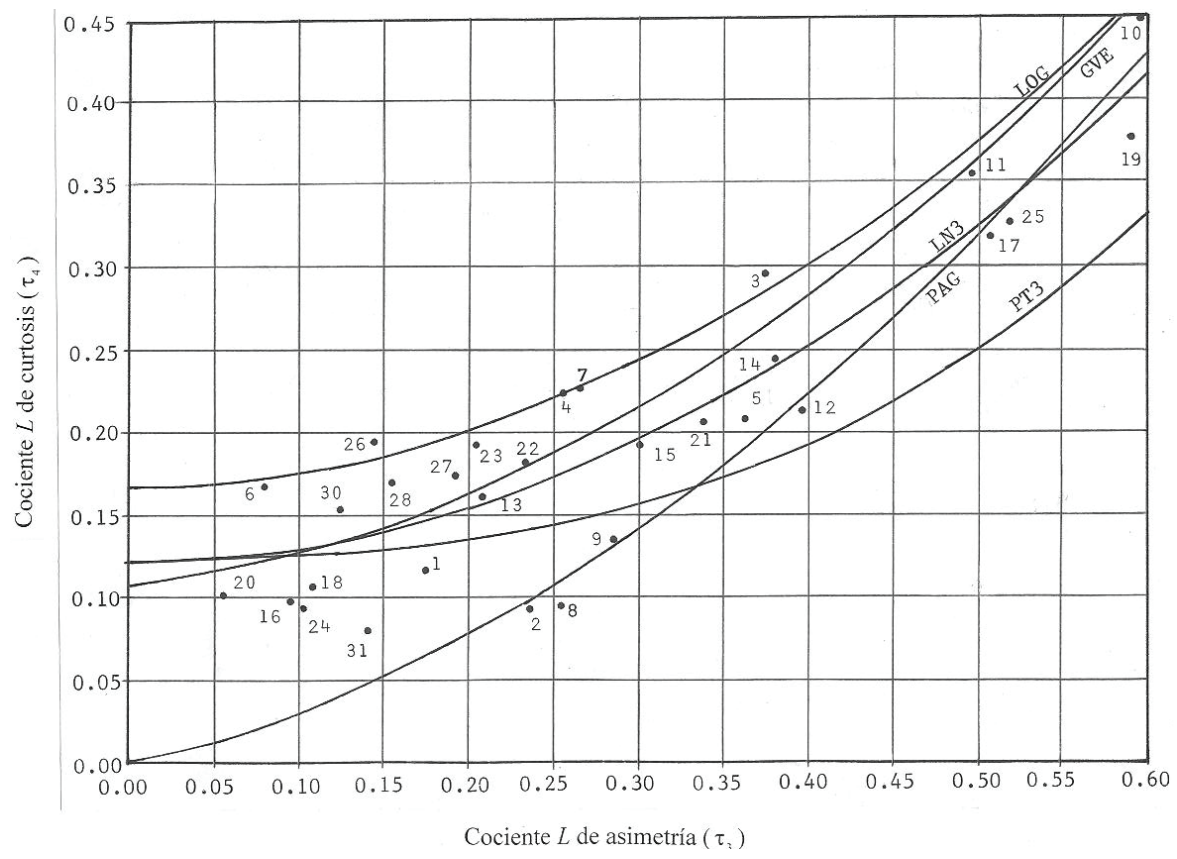




\section{Registros procesados}

Las referencias de procedencia de los 31 registros históricos que se usarán se pueden consultar en Campos (2001, 2002a, b), así como sus parámetros estadísticos insesgados. En cambio, en el cuadro 1 se presentan los valores de sus momentos y cocientes $L$, según las ecuaciones 1 a 7 . Los valores de los cocientes $\tau_{3}$ y $\tau_{4}$ de cada registro se llevaron al diagrama de momentos $L$ para dibujar puntos que por su cercanía a una cierta curva, definen la distribución de probabilidades más conveniente (figura 1).
Estos resultados están en la columna 2 del cuadro 2 y lógicamente sólo pueden ser los modelos: LOG, GVE, LN3, PT3 y PAG. Además se observa que únicamente los registros números 3, 4, 6, 7, 10, 23, 26 y 28 se aproximan a la curva de la distribución LOG y que los registros números 2, 8, 9 y 31 son los más alejados de ella.

La distribución LOG se aplicará a los 31 registros procesados, pero en el análisis de resultados se revisará con mayor acuciosidad estos dos grupos de registros.

Cuadro 1. Momentos y cocientes de momentos $L$ de los 31 registros históricos procesados

\begin{tabular}{|c|c|c|c|c|c|c|}
\hline Número de registro y nombre del río: & $n$ & $l_{1}$ & $l_{2}$ & $t_{2}$ & $t_{3}$ & $t_{4}$ \\
\hline 1. Ejemplo 6-3, Tabla 6.2. & 16 & 1704.375 & 455.875 & 0.26747 & 0.17662 & 0.11678 \\
\hline 2. Río Nackawic en 01AK007, Canadá & 21 & 55.824 & 12.880 & 0.23073 & 0.23555 & 0.09142 \\
\hline 3. Río Maury en Lexington, Virginia, USA & 26 & 328.638 & 101.761 & 0.30965 & 0.37397 & 0.29472 \\
\hline 4. Río Ouse en Skelton, Inglaterra & 28 & 351.250 & 51.718 & 0.14724 & 0.25466 & 0.22151 \\
\hline 5. Río Tana en Garissa, Kenia & 31 & 838.999 & 304.141 & 0.36250 & 0.36151 & 0.20923 \\
\hline 6. Río Irwell en Adelphi Weir, Inglaterra & 31 & 231.670 & 50.173 & 0.21657 & 0.08024 & 0.16833 \\
\hline 7. Río Cypress Creek en Houston, Texas, USA & 31 & 117.343 & 49.061 & 0.41810 & 0.26479 & 0.22365 \\
\hline 8. Río Nidd en Hunsingore, Inglaterra & 35 & 136.663 & 33.434 & 0.24465 & 0.25348 & 0.09261 \\
\hline 9. Río Valles en Santa Rosa, SLP, México & 36 & 789.445 & 326.194 & 0.41319 & 0.28267 & 0.13124 \\
\hline 10. Río Floyd en James, Iowa, USA & 39 & 191.736 & 115.072 & 0.60016 & 0.59523 & 0.44937 \\
\hline 11. Río Sinaloa en Jaina, Sinaloa, México & 40 & 1125.975 & 449.852 & 0.39952 & 0.49668 & 0.35404 \\
\hline 12. Río Guadalupe en Victoria, Texas, USA & 44 & 800.979 & 385.172 & 0.48088 & 0.39767 & 0.21139 \\
\hline 13. Río Manawatu, Nueva Zelanda & 45 & 1734.244 & 436.824 & 0.25188 & 0.20775 & 0.16068 \\
\hline 14. Río Saskatchewan en Edmonton, Canadá & 47 & 49.996 & 14.933 & 0.29869 & 0.38044 & 0.24498 \\
\hline 15. Río Santiago en Carrizal, Nayarit, México & 50 & 2699.780 & 722.653 & 0.26767 & 0.30074 & 0.19099 \\
\hline 16. Río Bow en Banff, Alberta, Canadá & 53 & 221.945 & 34.423 & 0.15510 & 0.09604 & 0.09866 \\
\hline 17. Río Fuerte en Huites, Sinaloa, México & 53 & 3176.434 & 1453.917 & 0.45772 & 0.50858 & 0.31946 \\
\hline 18. Río Clearwater en Kamiah, Idaho, USA & 55 & 1556.600 & 262.540 & 0.16866 & 0.10948 & 0.10513 \\
\hline $\begin{array}{l}\text { 19. Río San Rodrigo en Cerca del Moral } \\
\text { Coahuila, México }\end{array}$ & 55 & 327.800 & 223.791 & 0.68271 & 0.58924 & 0.37464 \\
\hline 20. Río Tennessee en Chattanooga, USA & 57 & 208.560 & 33.051 & 0.15847 & 0.05494 & 0.10148 \\
\hline 21. Río Waimakariri en Old Bridge, Nueva Zelanda & 57 & 1490.702 & 361.034 & 0.24219 & 0.33790 & 0.20694 \\
\hline 22. Río Piscataquis en Dover-Foxcroft, Maine, USA & 58 & 244.086 & 63.197 & 0.25891 & 0.23256 & 0.18047 \\
\hline 23. Río St. Marys en Stillwater, Nueva Escocia, Canadá & 59 & 409.578 & 78.535 & 0.19175 & 0.20427 & 0.19191 \\
\hline 24. Río Kentucky en Salvisa, USA & 66 & 1911.642 & 339.965 & 0.17784 & 0.01024 & 0.09361 \\
\hline 25. Río San Juan en El Cuchillo, Nuevo León, México & 67 & 1139.560 & 651.000 & 0.57127 & 0.51895 & 0.32703 \\
\hline 26. Río Harricana en Amos, Québec, Canadá & 69 & 191.317 & 26.121 & 0.13653 & 0.14525 & 0.19306 \\
\hline 27. Río Támesis en Teddington, Inglaterra & 85 & 319.529 & 66.927 & 0.20945 & 0.19039 & 0.17347 \\
\hline 28. Río Támesis en Kingston, Inglaterra & 113 & 324.487 & 64.349 & 0.19831 & 0.15436 & 0.16966 \\
\hline 29. Río Tampaón en Tamuín (niveles, m), SLP, México & 21 & 21.689 & 1.376 & 0.06346 & -0.15284 & 0.09797 \\
\hline 30. Precipitación máxima anual $(\mathrm{mm})$ en Baver, Suiza & 70 & 47.486 & 7.210 & 0.15183 & 0.12331 & 0.15185 \\
\hline $\begin{array}{l}\text { 31. Vel. máxima de viento }(\mathrm{km} / \mathrm{h}) \text { en } \\
\text { Shieffield, Inglaterra }\end{array}$ & 72 & 66.260 & 8.961 & 0.13524 & 0.14060 & 0.07917 \\
\hline
\end{tabular}




\section{Ajuste de la distribución Logística Generalizada mediante momentos $L$}

Este modelo probabilístico tiene tres parámetros: $\xi$ (ubicación), $\alpha$ (escala) y $k$ (forma). La variable $x$ fluctúa así: $-\infty<x \leq \xi+\alpha / k$ si $k>0$, de $-\infty<x<\infty$ si $k=0 \mathrm{y}$ de $\xi+\alpha / k \leq x<\infty$ si $k<0$. Incluye como caso especial a la distribución Logística cuando $k=0$, sus funciones de densidad y de distribución de probabilidades son (Hosking y Wallis, 1997):

$$
\begin{aligned}
& f(x)=\frac{\alpha^{-1} \cdot e^{-(1-k) y}}{\left(1+e^{-y}\right)^{2}} \\
& F(x)=\frac{1}{\left(1+e^{-y}\right)}
\end{aligned}
$$

donde $y=-k^{-1} \cdot \log [1-k \cdot(x-\xi) / \alpha]$ cuando $k \neq 0$

$$
y=(x-\xi) / \alpha \quad \text { cuando } k=0
$$

Las soluciones inversas son:

$$
\begin{array}{ll}
x(F)=\xi+\frac{\alpha}{k}\left\{1-\left[\frac{(1-F)}{F}\right]^{k}\right\} & \text { cuando } k \neq 0 \\
x(F)=\xi-\alpha \cdot \log \left[\frac{(1-F)}{F}\right] & \text { cuando } k=0
\end{array}
$$

donde $F$ es probabilidad de no excedencia. Las expresiones de los parámetros de ajuste son:

$$
\begin{aligned}
& k=-t_{3} \\
& \alpha=\frac{l_{2} \cdot \operatorname{sen}(\pi \cdot k)}{\pi \cdot k} \\
& \xi=l_{1}-\alpha\left(\frac{1}{k}-\frac{\pi}{\operatorname{sen}(\pi \cdot k)}\right)
\end{aligned}
$$

\section{Ajuste de la distribución Logística Generalizada mediante optimización numérica}

Las distribuciones general de valores extremos (GVE) y Log-Pearson tipo III (LP3) se ajustaron mediante optimización numérica para minimizar el error cuadrático medio y el error absoluto medio. Los resultados muestran (Campos, 2001; 2002b) similitud en las predicciones alcanzadas con cada enfoque, además de que se observa una correspondencia numérica entre los valores mínimos de tales errores que son alcanzados en cada registro. Debido a lo anterior, se consideró suficiente contrastar la distribución logística generalizada (LOG) minimizan- do únicamente el error cuadrático medio, usualmente conocido como error estándar de ajuste (EEA). Nuevamente, este proceso se realizó con base en el algoritmo de múltiples variables no restringidas de Rosenbrock (Kuester y Mize, 1973; Campos 2003), considera como variables a optimizar sus tres parámetros de ajuste, al igual que en el modelo GVE, cuyos valores iniciales fueron los del método de momentos L. En cambio, en la distribución LP3 tales variables de ajuste corresponden a los estadísticos logarítmicos, media, desviación estándar y coeficiente de asimetría corregido, es decir: $Y m$, Sy y $g_{c}$ (Campos, 2002b).

\section{Resultados y discusión}

Resumen de resultados

En el cuadro 2 para cada registro procesado hay seis renglones de resultados, los dos primeros proceden de Campos (2001) y corresponden a los parámetros de ajuste $(u, \alpha, k), E E A$ y predicciones con periodos de retorno 10, 25, 50, 100, 500, 1000 y 10000 años obtenidas con la distribución GVE, con uno de los cuatro métodos de ajuste, el que condujo al EEA mínimo y con el ajuste mediante optimización numérica (OPN), indicando, en la columna 8 del cuadro 2, los números de etapas y evaluaciones de la función objetivo (EEA) realizadas; en la columna 3 se indica el método de ajuste (MA): momentos $L$ (MOL), optimización numérica (OPN), máxima verosimilitud (MMV), sextiles (SEX), momentos en el dominio logarítmico (MML), mezcla de momentos (MMM), máxima entropía (MME) y promedios diversos (MPD). Los siete periodos de retorno citados, cubren los diversos dimensionamientos y/o revisiones hidrológicas de las obras hidráulicas.

De manera similar, en los renglones 3 y 4 de cada registro se presentan idénticos resultados para la distribución LP3, pero utilizando alguno de sus seis métodos de ajuste y el de OPN. Finalmente en los renglones 5 y 6 de cada registro están los resultados de la distribución LOG, en este caso, ajustada mediante el método de momentos $L$ (ecuaciones 16 a 20) y de OPN.

El algoritmo de OPN únicamente falló en los registros 19, 25 y 31 al ajustar la distribución LOG, lo cual se corrigió limitando el número de etapas permitido a la última en que se tenían resultados consistentes.

\section{Análisis global de resultados}

En el cuadro 2 se presenta un contraste global de los resultados obtenidos para los tres modelos probabilísticos probados (GVE, LP3 y LOG), concluyéndose: 
1) En todos los casos, con el método de optimización numérica (OPN), el EEA es reducido más allá del mínimo obtenido con los procedimientos estadísticos;

2) Las tres distribuciones conducen a valores del EEA del mismo orden de magnitud, ya sea a través de los métodos estadísticos o con el de OPN;

3) Lo mismo se puede decir para sus parámetros de ajuste, los cuales no cambian radicalmente con el método de ajuste;

4) Respecto a las predicciones, en general, sus magnitudes son bastante coincidentes en periodos de retorno bajos (<50 años), incluso en ciertos registros hasta los periodos de retorno elevados ( $>1,000$ años), tal es el caso de los registros 2, 6, 9, 16, 18, 20, 22, 26, 27, 28 y 31;

5) En registros que presentan valores extremos dispersos (outliers), sus predicciones en los periodos de retorno altos (> 1,000 años) varían notablemente, como en los registros 10, 11, 12, 17 y 25.

Las observaciones globales del cuadro 2 destacan que la distribución LP3 es mucho menos flexible en la búsqueda del EEA mínimo a través del método de OPN, como se muestra por la similitud entre sus predicciones en los periodos de retorno de 1,000 y 10,000 años, las cuales casi siempre resultaron similares con el método estadístico y el de OPN. Lo contrario ocurre con las distribuciones GVE y LOG, las cuales casi siempre conducen a predicciones bastante dispersas en los periodos de retorno citados, con el método estadístico de ajuste y con la OPN.

\section{Análisis específico de resultados}

Para los ocho registros donde es recomendable la distribución LOG se observa (cuadro 2) que sus EEA mínimos son plenamente coincidentes con los de los modelos GVE y LP3. También son similares las predic- ciones de los tres modelos en los periodos de retorno reducidos, pero en general resultan superiores las de la distribución LOG en relación con las otras dos, en los periodos de retorno de 1000 y 10000 años. Cuando el modelo LOG no es el más conveniente, por ejemplo en los registros 2, 8, 9 y 31, sus EEA mínimos son mayores que los obtenidos con las distribuciones GVE y LP3 y también sus predicciones resultan superiores en los periodos de retorno elevados.

\section{Conclusiones}

La aplicación de las tres distribuciones de probabilidad contrastadas (general de valores extremos, Log-Pearson tipo III y logística generalizada) es recomendable en los análisis probabilísticos de crecientes y de otros datos hidrológicos extremos, debido a la consistencia o similitud numérica que presentan todas sus predicciones en los periodos de retorno reducidos ( $<50$ años), sin importar el método de ajuste. La distribución logística generalizada ofrece una opción probabilística adecuada o conveniente a los registros con valores grandes del cociente $L$ de curtosis $\left(\tau_{4}\right)$, tanto en los análisis probabilísticos locales como regionales. En registros que presenten valores extremos dispersos (outliers), la distribución logística generalizada, permitirá un ajuste muy bueno a los datos, por medio del método de optimización numérica, pero debido a ello sus predicciones en los periodos de retorno elevados serán muy grandes.

\section{Anexo}

Cuadro 2. Resultados del mejor método de ajuste $(<E E A)$ y de la optimización numérica para las distribuciones GVE, Log-Pearson tipo III y Logística Generalizada en los 31 registros históricos procesados

\begin{tabular}{|c|c|c|c|c|c|c|c|c|c|c|c|c|c|c|}
\hline \multirow[b]{2}{*}{ NR } & \multirow[b]{2}{*}{ DA } & \multirow[b]{2}{*}{ MA } & \multicolumn{3}{|c|}{ Parámetros o variables de ajuste } & \multirow{2}{*}{$\begin{array}{c}E E A \\
\left(\mathrm{~m}^{3} / \mathrm{s}\right)\end{array}$} & \multirow{2}{*}{$\begin{array}{l}\text { (etapas) } \\
\text { No.eval. }\end{array}$} & \multicolumn{7}{|c|}{ Periodos de retorno en años } \\
\hline & & & $u$ ó Ym & $\alpha$ ó $S y$ & $k$ ó $g_{c}$ & & & 10 & 25 & 50 & 100 & 500 & 1000 & 10000 \\
\hline 1 & & MOL & 1354.333 & 651.656 & -0.01045 & 152.8 & - & 2838 & 3474 & 3950 & 4425 & 5538 & 6022 & 7654 \\
\hline 1 & & OPN & 1314.343 & 710.061 & -0.04934 & 118.7 & (6) 77 & 3004 & 3775 & 4370 & 4981 & 6477 & 7158 & 9593 \\
\hline 1 & & MML & 7.33750 & 0.47700 & -0.16191 & 164.1 & - & 2805 & 3448 & 3927 & 4406 & 5532 & 6026 & 7715 \\
\hline 1 & & OPN & 7.33095 & 0.54632 & -0.05204 & 115.8 & (5) 59 & 3063 & 3934 & 4620 & 5333 & 7116 & 7943 & 10979 \\
\hline 1 & PT3 & MOL & 1573.953 & 432.840 & -0.17662 & 187.1 & - & 2736 & 3419 & 3996 & 4641 & 6466 & 7423 & 11590 \\
\hline 1 & & OPN & 1585.836 & 495.145 & -0.22334 & 127.0 & (9) 96 & 2990 & 3877 & 4656 & 5556 & 8248 & 9737 & 16711 \\
\hline 2 & & MOL & 44.431 & 16.805 & -0.10008 & 5.0 & - & 87 & 108 & 125 & 143 & 189 & 212 & 299 \\
\hline 2 & & OPN & 44.284 & 18.678 & -0.11427 & 4.0 & (5) 64 & 92 & 116 & 136 & 157 & 213 & 241 & 349 \\
\hline 2 & & MML & 3.94563 & 0.39843 & 0.25293 & 5.0 & - & 87 & 107 & 124 & 141 & 184 & 205 & 285 \\
\hline 2 & & OPN & 3.95179 & 0.44325 & 0.03372 & 4.0 & (2) 32 & 92 & 114 & 130 & 148 & 190 & 209 & 280 \\
\hline 2 & PAG & MOL & 50.968 & 11.736 & -0.23555 & 5.8 & - & 85 & 107 & 126 & 148 & 216 & 255 & 437 \\
\hline 2 & & OPN & 51.399 & 13.045 & -0.29079 & 4.3 & (3) 29 & 92 & 120 & 146 & 177 & 280 & 341 & 660 \\
\hline 3 & & MOL & 228.506 & 102.689 & -0.29557 & 73.8 & - & 557 & 775 & 982 & 1234 & 2061 & 2557 & 5166 \\
\hline 3 & & OPN & 222.626 & 103.217 & -0.48721 & 34.4 & (5) 35 & 645 & 1017 & 1429 & 2003 & 4384 & 6142 & 18836 \\
\hline
\end{tabular}




\begin{tabular}{|c|c|c|c|c|c|c|c|c|c|c|c|c|c|c|}
\hline \multirow[b]{2}{*}{ NR } & \multirow[b]{2}{*}{ DA } & \multirow[b]{2}{*}{ MA } & \multicolumn{3}{|c|}{ Parámetros o variables de ajuste } & \multirow{2}{*}{$\begin{array}{c}E E A \\
\left(\mathrm{~m}^{3} / \mathrm{s}\right)\end{array}$} & \multirow{2}{*}{$\begin{array}{l}\text { (etapas) } \\
\text { No.eval. }\end{array}$} & \multicolumn{7}{|c|}{ Periodos de retorno en años } \\
\hline & & & $u$ ó Ym & $\alpha$ ó $S y$ & $k$ ó $g_{c}$ & & & 10 & 25 & 50 & 100 & 500 & 1000 & 10000 \\
\hline 3 & & MMM & 5.64795 & 0.53160 & 0.60848 & 69.3 & - & 563 & 733 & 870 & 1017 & 1401 & 1587 & 2306 \\
\hline 3 & & OPN & 5.68859 & 0.58065 & 0.28644 & 34.1 & (12) 123 & 631 & 863 & 1063 & 1289 & 1931 & 2267 & 3707 \\
\hline 3 & LOG & MOL & 270.220 & 79.914 & -0.37397 & 77.9 & - & 543 & 758 & 973 & 1248 & 2238 & 2885 & 6750 \\
\hline 3 & & OPN & 256.736 & 73.615 & -0.65487 & 31.4 & (14) 130 & 618 & 1045 & 1582 & 2423 & 6717 & 10499 & 46943 \\
\hline 4 & & MOL & 304.603 & 65.360 & -0.12830 & 21.2 & - & 475 & 563 & 636 & 714 & 926 & 1031 & 1456 \\
\hline 4 & & OPN & 302.703 & 70.727 & -0.18816 & 15.1 & (3) 36 & 501 & 613 & 710 & 820 & 1137 & 1306 & 2053 \\
\hline 4 & & MMM & 5.82925 & 0.25088 & 0.98022 & 21.1 & - & 471 & 535 & 582 & 628 & 737 & 784 & 946 \\
\hline 4 & & OPN & 5.83748 & 0.28246 & 0.16921 & 15.1 & (3) 38 & 495 & 571 & 628 & 685 & 820 & 880 & 1089 \\
\hline 4 & LOG & MOL & 330.268 & 46.375 & -0.25466 & 23.1 & - & 467 & 557 & 639 & 735 & 1034 & 1205 & 2049 \\
\hline 4 & & OPN & 329.236 & 51.012 & -0.33106 & 14.7 & (2) 25 & 494 & 616 & 734 & 881 & 1380 & 1692 & 3426 \\
\hline 5 & & MMV & 524.470 & 277.582 & -0.43927 & 104.4 & - & 1591 & 2468 & 3400 & 4660 & 9576 & 13026 & 36002 \\
\hline 5 & & OPN & 530.981 & 307.901 & -0.42979 & 83.1 & (7) 85 & 1699 & 2647 & 3647 & 4988 & 10165 & 13760 & 37329 \\
\hline 5 & & MML & 6.53361 & 0.62233 & 0.48521 & 140.6 & - & 1564 & 2252 & 2888 & 3646 & 6003 & 7343 & 13795 \\
\hline 5 & & OPN & 6.55752 & 0.66673 & 0.12861 & 85.4 & (12) 128 & 1668 & 2330 & 2901 & 3542 & 5339 & 6266 & 10153 \\
\hline 5 & PAG & MOL & 669.452 & 242.847 & -0.36151 & 173.0 & - & 1484 & 2117 & 2741 & 3535 & 6345 & 8156 & 18757 \\
\hline 5 & & OPN & 661.434 & 250.600 & -0.50781 & 82.3 & (7) 95 & 1674 & 2646 & 3729 & 5258 & 11740 & 16630 & 53191 \\
\hline 6 & & MMV & 193.794 & 77.343 & 0.09530 & 19.9 & - & 350 & 407 & 446 & 482 & 556 & 585 & 668 \\
\hline 6 & & OPN & 194.915 & 80.472 & 0.05088 & 18.2 & (6) 82 & 366 & 432 & 480 & 525 & 623 & 663 & 786 \\
\hline 6 & & MME & 5.36830 & 0.40380 & 0.03810 & 18.6 & - & 360 & 435 & 492 & 550 & 689 & 751 & 969 \\
\hline 6 & & OPN & 5.37352 & 0.41913 & -0.03141 & 18.0 & (7) 97 & 368 & 447 & 507 & 567 & 710 & 774 & 998 \\
\hline 6 & LOG & MOL & 225.069 & 49.643 & -0.08024 & 19.7 & - & 344 & 405 & 452 & 501 & 625 & 683 & 902 \\
\hline 6 & & OPN & 222.698 & 53.671 & -0.15198 & 16.0 & (4) 60 & 363 & 442 & 508 & 580 & 777 & 878 & 1301 \\
\hline 7 & & MOL & 72.670 & 60.935 & -0.14309 & 20.9 & - & 234 & 320 & 391 & 469 & 683 & 791 & 1238 \\
\hline 7 & & OPN & 69.492 & 61.054 & -0.27199 & 11.6 & (17) 142 & 259 & 381 & 494 & 629 & 1062 & 1314 & 2593 \\
\hline 7 & & MPD & 4.40899 & 0.93576 & -0.75429 & 21.2 & - & 269 & 406 & 527 & 665 & 1056 & 1257 & 2088 \\
\hline 7 & & OPN & 4.51265 & 0.80294 & 0.01951 & 12.9 & (15) 157 & 255 & 374 & 479 & 598 & 939 & 1117 & 1872 \\
\hline 7 & LOG & MOL & 96.701 & 43.595 & -0.26479 & 22.3 & - & 227 & 314 & 394 & 488 & 785 & 957 & 1819 \\
\hline 7 & & OPN & 93.038 & 45.880 & -0.39338 & 10.1 & (29) 254 & 253 & 384 & 516 & 687 & 1320 & 1742 & 4345 \\
\hline 8 & & MOL & 106.538 & 42.321 & -0.12686 & 9.7 & - & 217 & 273 & 320 & 371 & 507 & 574 & 846 \\
\hline 8 & & OPN & 105.907 & 38.949 & -0.23170 & 9.6 & (1) 9 & 221 & 291 & 353 & 426 & 647 & 771 & 1358 \\
\hline 8 & & MML & 4.82973 & 0.41880 & 0.40433 & 9.7 & - & 217 & 275 & 323 & 375 & 516 & 586 & 870 \\
\hline 8 & & OPN & 4.83029 & 0.46171 & 0.02776 & 7.4 & (3) 59 & 227 & 282 & 326 & 370 & 481 & 532 & 718 \\
\hline 8 & PAG & MOL & 123.158 & 30.011 & -0.25348 & 11.8 & - & 211 & 270 & 322 & 384 & 577 & 687 & 1227 \\
\hline 8 & & OPN & 124.751 & 32.826 & -0.27250 & 9.1 & (3) 40 & 224 & 291 & 352 & 426 & 659 & 795 & 1486 \\
\hline 9 & & MMV & 472.973 & 355.577 & -0.27754 & 89.5 & - & 1584 & 2305 & 2976 & 3785 & 6379 & 7905 & 15700 \\
\hline 9 & & OPN & 488.439 & 431.984 & -0.17485 & 61.4 & (8) 83 & 1680 & 2340 & 2906 & 3540 & 5340 & 6284 & 10382 \\
\hline 9 & & MPD & 6.36414 & 0.84715 & -0.60666 & 84.5 & - & 1701 & 2483 & 3160 & 3916 & 6007 & 7064 & 11339 \\
\hline 9 & & OPN & 6.36414 & 0.94710 & -0.12772 & 55.0 & (1) 9 & 1925 & 2921 & 3807 & 4815 & 7677 & 9156 & 15279 \\
\hline 9 & PAG & MOL & 643.642 & 284.979 & -0.28267 & 112.0 & - & 1512 & 2111 & 2664 & 3031 & 5473 & 6738 & 13256 \\
\hline 9 & & OPN & 643.642 & 313.477 & -0.28267 & 82.3 & (1) 9 & 1598 & 2258 & 2867 & 3599 & 5956 & 7348 & 14517 \\
\hline 10 & & MMV & 68.104 & 61.520 & -0.69011 & 155.1 & - & 400 & 789 & 1296 & 2111 & 6471 & 10457 & 51308 \\
\hline 10 & & OPN & 64.609 & 28.076 & -1.19579 & 62.7 & (10) 94 & 387 & 1117 & 2536 & 5791 & 39629 & 90785 & $1.4 \cdot 106$ \\
\hline 10 & & MML & 4.62216 & 1.06880 & 0.41776 & 170.0 & - & 415 & 763 & 1153 & 1694 & 3837 & 5329 & 14799 \\
\hline 10 & & OPN & 4.56416 & 1.17500 & 0.29390 & 71.3 & (5) 36 & 446 & 841 & 1287 & 1904 & 4332 & 6004 & 16361 \\
\hline 10 & LOG & MOL & 97.204 & 58.803 & -0.59523 & 193.8 & - & 364 & 653 & 1000 & 1521 & 3986 & 6026 & 23743 \\
\hline 10 & & OPN & 80.8544 & 29.823 & -1.17626 & 63.3 & (18) 170 & 392 & 1121 & 2522 & 5697 & 37875 & 85625 & $1.3 \cdot 106$ \\
\hline 11 & & MMV & 653.307 & 326.064 & -0.53163 & 435.4 & - & 2069 & 3399 & 4922 & 7116 & 16724 & 24165 & 82090 \\
\hline 11 & & OPN & 647.115 & 217.322 & -0.86285 & 239.4 & (24) 197 & 2151 & 4374 & 7696 & 13730 & 54049 & 98018 & 712207 \\
\hline 11 & & MML & 6.77948 & 0.64206 & 1.12598 & 464.7 & - & 2069 & 3315 & 4662 & 6498 & 13755 & 18888 & 53440 \\
\hline 11 & & OPN & 6.83418 & 0.69789 & 0.42785 & 245.6 & (17) 176 & 2328 & 3471 & 4551 & 5859 & 10031 & 12452 & 24405 \\
\hline 11 & GVE & MOL & 800.681 & 288.286 & -0.49667 & 527.8 & - & 1949 & 3034 & 4231 & 5908 & 12921 & 18150 & 56505 \\
\hline 11 & & OPN & 770.339 & 204.980 & -0.88059 & 241.6 & (15) 155 & 2149 & 4360 & 7704 & 13851 & 55856 & 102475 & 775362 \\
\hline 12 & & MMV & 389.809 & 317.042 & -0.50729 & 254.6 & - & 1722 & 2931 & 4289 & 6211 & 14380 & 20544 & 66581 \\
\hline 12 & & OPN & 443.176 & 288.938 & -0.58068 & 245.1 & (16) 164 & 1784 & 3133 & 4742 & 7140 & 18305 & 27412 & 104528 \\
\hline 12 & & MMV & 6.27776 & 0.92300 & 0.13929 & 286.3 & - & 1740 & 2700 & 3589 & 4638 & 7804 & 9537 & 17309 \\
\hline 12 & & OPN & 6.39346 & 0.81139 & 0.17226 & 248.8 & (13) 162 & 1713 & 2594 & 3410 & 4379 & 7345 & 8996 & 16629 \\
\hline 12 & PAG & MOL & 567.972 & 292.512 & -0.39767 & 336.2 & - & 1595 & 2436 & 3290 & 4406 & 8534 & 11300 & 28491 \\
\hline
\end{tabular}




\begin{tabular}{|c|c|c|c|c|c|c|c|c|c|c|c|c|c|c|}
\hline \multirow[b]{2}{*}{ NR } & \multirow[b]{2}{*}{ DA } & \multirow[b]{2}{*}{ MA } & \multicolumn{3}{|c|}{ Parámetros o variables de ajuste } & \multirow{2}{*}{$\begin{array}{c}E E A \\
\left(\mathrm{~m}^{3} / \mathrm{s}\right)\end{array}$} & \multirow{2}{*}{$\begin{array}{l}\text { (etapas) } \\
\text { No.eval. }\end{array}$} & \multicolumn{7}{|c|}{ Periodos de retorno en años } \\
\hline & & & $u$ ó Ym & $\alpha$ ó $S y$ & $k$ ó $g_{c}$ & & & 10 & 25 & 50 & 100 & 500 & 1000 & 10000 \\
\hline 12 & & OPN & 605.449 & 328.265 & -0.48604 & 255.8 & (3) 30 & 1895 & 3095 & 4408 & 6233 & 13764 & 19315 & 59312 \\
\hline 13 & & MOL & 1360.919 & 595.786 & -0.05831 & 104.6 & - & 2794 & 3456 & 3971 & 4504 & 5822 & 6428 & 8625 \\
\hline 13 & & OPN & 1336.472 & 604.967 & -0.12056 & 70.6 & (10) 125 & 2900 & 3698 & 4351 & 5056 & 6932 & 7858 & 11550 \\
\hline 13 & & MMV & 7.35932 & 0.45244 & 0.16003 & 89.2 & - & 2807 & 3483 & 4005 & 4543 & 5868 & 6476 & 8683 \\
\hline 13 & & OPN & 7.36468 & 0.47102 & 0.02658 & 74.0 & (17) 171 & 2890 & 3618 & 4184 & 4771 & 6227 & 6900 & 9364 \\
\hline 13 & LN3 & MOL & 1588.111 & 406.465 & -0.20775 & 114.3 & - & 2720 & 3418 & 4023 & 4714 & 6744 & 7847 & 12889 \\
\hline 13 & & OPN & 1584.220 & 434.086 & -0.25198 & 66.2 & (7) 108 & 2858 & 3699 & 4455 & 5345 & 8104 & 9680 & 17404 \\
\hline 14 & & MMV & 34716 & 13.742 & -0.40480 & 4.6 & - & 85 & 125 & 166 & 219 & 421 & 557 & 1413 \\
\hline 14 & & OPN & 34.777 & 13.907 & -0.45370 & 3.0 & (7) 74 & 89 & 135 & 184 & 251 & 518 & 708 & 2005 \\
\hline 14 & & MML & 3.77705 & 0.49605 & 0.85605 & 6.6 & - & 85 & 118 & 150 & 188 & 309 & 381 & 745 \\
\hline 14 & & OPN & 3.79532 & 0.53169 & 0.20937 & 3.1 & (7) 78 & 89 & 117 & 141 & 166 & 236 & 271 & 411 \\
\hline 14 & LN3 & MOL & 41.296 & 11.623 & -0.38044 & 7.8 & - & 81 & 113 & 145 & 186 & 336 & 434 & 1026 \\
\hline 14 & & OPN & 41.308 & 12.602 & -0.47430 & 2.9 & (12) 126 & 90 & 135 & 183 & 250 & 521 & 718 & 2111 \\
\hline 15 & & SEX & 2003.078 & 831.726 & -0.21273 & 267.4 & - & 4404 & 5814 & 7060 & 8496 & 12756 & 15087 & 25828 \\
\hline 15 & & OPN & 2026.598 & 935.595 & -0.17413 & 232.5 & (6) 68 & 4604 & 6031 & 7253 & 8624 & 12507 & 14542 & 23365 \\
\hline 15 & & MML & 7.79066 & 0.46651 & 0.30729 & 254.4 & - & 4451 & 5736 & 6798 & 7953 & 11059 & 12607 & 18866 \\
\hline 15 & & OPN & 7.79009 & 0.50601 & 0.03126 & 223.4 & (7) 126 & 4626 & 5892 & 6892 & 7939 & 10581 & 11820 & 16438 \\
\hline 15 & LN3 & MOL & 2357.902 & 619.836 & -0.30074 & 303.7 & - & 4288 & 5657 & 6940 & 8505 & 13648 & 16748 & 33184 \\
\hline 15 & & OPN & 2433.709 & 702.022 & -0.27764 & 258.1 & (5) 68 & 4559 & 6016 & 7355 & 8961 & 14095 & 17110 & 32518 \\
\hline 16 & & MOL & 195.995 & 54.827 & 0.11901 & 4.7 & - & 304 & 342 & 367 & 390 & 437 & 454 & 503 \\
\hline 16 & & OPN & 196.590 & 56.643 & 0.12102 & 4.2 & (4) 86 & 308 & 347 & 373 & 396 & 444 & 462 & 511 \\
\hline 16 & & MML & 5.36596 & 0.27474 & -0.14776 & 5.3 & - & 303 & 341 & 368 & 394 & 450 & 473 & 546 \\
\hline 16 & & OPN & 5.36446 & 0.29216 & -0.04168 & 4.1 & (3) 52 & 310 & 355 & 387 & 418 & 488 & 518 & 618 \\
\hline 16 & РT3 & MOL & 216.532 & 33.904 & -0.09604 & 6.9 & - & 300 & 343 & 377 & 412 & 505 & 549 & 719 \\
\hline 16 & & OPN & 216.972 & 35.451 & -0.10079 & 6.3 & (4) 61 & 304 & 350 & 386 & 424 & 523 & 571 & 755 \\
\hline 17 & & MMV & 1628.807 & 996.848 & -0.57129 & 836.9 & - & 6195 & 10732 & 16097 & 24045 & 60616 & 90152 & 336240 \\
\hline 17 & & OPN & 1616.945 & 1421.410 & -0.44506 & 675.4 & (20) 276 & 7118 & 11683 & 16557 & 23166 & 49158 & 67510 & 190924 \\
\hline 17 & & MML & 7.73260 & 0.76133 & 0.85203 & 815.7 & - & 6290 & 10492 & 15061 & 21301 & 45851 & 63053 & 175998 \\
\hline 17 & & OPN & 7.68163 & 0.95060 & 0.02212 & 611.7 & (10) 150 & 7337 & 11533 & 15458 & 20126 & 34382 & 42580 & 78020 \\
\hline 17 & PAG & MOL & 2106.257 & 909.645 & -0.50858 & 1085.8 & - & 5786 & 9322 & 13263 & 18830 & 42460 & 60302 & 193860 \\
\hline 17 & & OPN & 2195.905 & 1188.785 & -0.50726 & 706.0 & (15) 153 & 6996 & 11601 & 16727 & 23962 & 54619 & 77735 & 250383 \\
\hline 18 & & MOL & 1354.106 & 411.226 & 0.09683 & 61.4 & - & 2186 & 2485 & 2690 & 2881 & 3274 & 3425 & 3860 \\
\hline 18 & & OPN & 1346.534 & 417.847 & 0.05730 & 55.3 & (11) 98 & 2229 & 2568 & 2808 & 3036 & 3531 & 3730 & 4337 \\
\hline 18 & & MML & 7.30675 & 0.29893 & -0.05445 & 60.5 & - & 2181 & 2501 & 2730 & 2953 & 3457 & 3671 & 4380 \\
\hline 18 & & OPN & 7.30704 & 0.31356 & -0.01055 & 54.8 & (4) 39 & 2226 & 2578 & 2834 & 3086 & 3664 & 3913 & 4755 \\
\hline 18 & PT3 & MOL & 1509.599 & 257.393 & -0.10948 & 64.9 & - & 2149 & 2488 & 2759 & 3047 & 3800 & 4167 & 5603 \\
\hline 18 & & OPN & 1504.580 & 268.004 & -0.13962 & 56.7 & (6) 86 & 2194 & 2577 & 2890 & 3231 & 4155 & 4620 & 6530 \\
\hline 19 & & SEX & 85.404 & 126.713 & -0.59493 & 218.2 & - & 685 & 1301 & 2043 & 3160 & 8458 & 12845 & 50915 \\
\hline 19 & & OPN & 78.052 & 197.718 & -0.52828 & 152.5 & (30) 257 & 933 & 1732 & 2644 & 3956 & 9676 & 14089 & 48253 \\
\hline 19 & & MML & 4.78295 & 1.48007 & 0.10623 & 160.8 & - & 807 & 1681 & 2718 & 4203 & 10282 & 14564 & 41466 \\
\hline 19 & & OPN & 4.92462 & 1.59587 & -0.06368 & 132.6 & (5) 46 & 1050 & 2171 & 3458 & 5242 & 12065 & 16576 & 42118 \\
\hline 19 & LN3 & MOL & 145.161 & 116.174 & -0.58924 & 235.1 & - & 668 & 1231 & 1901 & 2904 & 7615 & 11490 & 44792 \\
\hline 19 & & OPN & 159.423 & 126.657 & -0.70133 & 169.5 & (3) 21 & 822 & 1656 & 2746 & 4511 & 14071 & 22909 & 115314 \\
\hline 20 & & MOL & 185.436 & 55.216 & 0.18829 & 7.8 & - & 287 & 318 & 338 & 355 & 388 & 388 & 399 \\
\hline 20 & & OPN & 184.502 & 56.305 & 0.16552 & 7.4 & (7) 89 & 290 & 324 & 346 & 366 & 403 & 416 & 451 \\
\hline 20 & & MMM & 5.30009 & 0.29080 & -0.43105 & 7.8 & - & 290 & 331 & 360 & 388 & 451 & 478 & 566 \\
\hline 20 & & OPN & 5.29866 & 0.30391 & -0.07483 & 7.4 & (5) 74 & 295 & 338 & 369 & 399 & 467 & 496 & 591 \\
\hline 20 & GVE & MOL & 205.577 & 32.887 & -0.05494 & 8.0 & - & 282 & 320 & 348 & 378 & 449 & 482 & 600 \\
\hline 20 & & OPN & 205.398 & 34.361 & -0.06724 & 7.5 & (3) 49 & 287 & 327 & 358 & 390 & 470 & 507 & 644 \\
\hline 21 & & MMV & 1136.232 & 375.284 & -0.29940 & 102.7 & - & 2342 & 3149 & 3914 & 4852 & 7937 & 9797 & 19636 \\
\hline 21 & & OPN & 1138.408 & 425.841 & -0.25562 & 83.6 & (1) 9 & 2434 & 3246 & 3989 & 4872 & 7628 & 9210 & 17014 \\
\hline 21 & & MML & 7.21845 & 0.40700 & 0.77811 & 111.8 & - & 2345 & 3061 & 3691 & 4413 & 6534 & 7686 & 12929 \\
\hline 21 & & OPN & 7.22440 & 0.43891 & 0.13107 & 79.6 & (8) 96 & 2421 & 3018 & 3487 & 3977 & 5213 & 5794 & 7968 \\
\hline 21 & LN3 & MOL & 1301.039 & 296.947 & -0.33790 & 134.3 & - & 2269 & 2994 & 3696 & 4574 & 7593 & 9489 & 20167 \\
\hline 21 & & OPN & 1318.203 & 332.770 & -0.34981 & 93.0 & (10) 118 & 2419 & 3258 & 4079 & 5114 & 8726 & 11023 & 24219 \\
\hline 22 & & MOL & 188.371 & 82.858 & -0.09563 & 13.9 & - & 396 & 498 & 580 & 667 & 891 & 999 & 1412 \\
\hline 22 & & OPN & 186.696 & 90.853 & -0.09092 & 11.5 & (5) 30 & 414 & 524 & 612 & 706 & 946 & 1060 & 1496 \\
\hline
\end{tabular}




\begin{tabular}{|c|c|c|c|c|c|c|c|c|c|c|c|c|c|c|}
\hline \multirow[b]{2}{*}{ NR } & \multirow[b]{2}{*}{ DA } & \multirow[b]{2}{*}{ MA } & \multicolumn{3}{|c|}{ Parámetros o variables de ajuste } & \multirow{2}{*}{$\begin{array}{c}E E A \\
\left(\mathrm{~m}^{3} / \mathrm{s}\right)\end{array}$} & \multirow{2}{*}{$\begin{array}{l}\text { (etapas) } \\
\text { No.eval. }\end{array}$} & \multicolumn{7}{|c|}{ Periodos de retorno en años } \\
\hline & & & $u$ ó Ym & $\alpha$ ó $S y$ & $k$ ó $g_{c}$ & & & 10 & 25 & 50 & 100 & 500 & 1000 & 10000 \\
\hline 22 & & MMV & 5.39146 & 0.46596 & 0.11666 & 12.7 & - & 399 & 498 & 575 & 654 & 850 & 940 & 1268 \\
\hline 22 & & OPN & 5.39286 & 0.48836 & 0.00813 & 11.0 & (9) 97 & 411 & 518 & 601 & 687 & 902 & 1001 & 1365 \\
\hline 22 & GVE & MOL & 220.547 & 57.723 & -0.23256 & 16.7 & - & 386 & 492 & 586 & 695 & 1025 & 1209 & 2086 \\
\hline 22 & & OPN & 220.547 & 63.495 & -0.23256 & 13.5 & (1) 9 & 403 & 519 & 623 & 742 & 1105 & 1308 & 2273 \\
\hline 23 & & MOL & 342.811 & 107.694 & -0.05301 & 25.0 & - & 600 & 718 & 810 & 904 & 1135 & 1241 & 1621 \\
\hline 23 & & OPN & 329.147 & 117.360 & -0.10033 & 21.6 & (6) 91 & 625 & 772 & 890 & 1015 & 1341 & 1498 & 2106 \\
\hline 23 & & MMM & 5.95790 & 0.33555 & 0.34764 & 23.8 & - & 596 & 701 & 779 & 857 & 1041 & 1123 & 1406 \\
\hline 23 & & OPN & 5.96422 & 0.34782 & 0.10830 & 18.4 & (9) 113 & 610 & 725 & 812 & 899 & 1110 & 1205 & 1542 \\
\hline 23 & LOG & MOL & 383.728 & 73.255 & -0.20427 & 23.7 & - & 587 & 712 & 819 & 942 & 1301 & 1495 & 2378 \\
\hline 23 & & OPN & 381.324 & 76.095 & -0.27097 & 14.5 & (6) 75 & 610 & 765 & 907 & 1076 & 1613 & 1925 & 3507 \\
\hline 24 & & MOL & 1695.071 & 594.815 & 0.26634 & 51.2 & & 2702 & 2976 & 3138 & 3272 & 3502 & 3574 & 3736 \\
\hline 24 & & OPN & 1687.454 & 607.700 & 0.25752 & 48.7 & (5) 56 & 2725 & 3012 & 3183 & 3326 & 3571 & 3649 & 3827 \\
\hline 24 & & MPD & 7.50196 & 0.34210 & -0.78476 & 54.6 & - & 2793 & 3246 & 3571 & 3887 & 4598 & 4899 & 5890 \\
\hline 24 & & OPN & 7.49805 & 0.35951 & -0.14677 & 50.0 & (6) 93 & 2842 & 3324 & 3670 & 4008 & 4769 & 5082 & 6156 \\
\hline 24 & PT3 & MOL & 1905.918 & 339.906 & -0.01024 & 65.6 & - & 2661 & 3004 & 3256 & 3505 & 4086 & 4339 & 5189 \\
\hline 24 & & OPN & 1904.117 & 351.197 & -0.01525 & 62.6 & (4) 65 & 2689 & 3048 & 3312 & 3576 & 4193 & 4462 & 5377 \\
\hline 25 & & MMV & 425.699 & 403.393 & -0.67640 & 286.2 & - & 2562 & 5019 & 8180 & 13221 & 39714 & 63594 & 302482 \\
\hline 25 & & OPN & 501.829 & 501.411 & -0.55541 & 206.3 & (7) 92 & 2749 & 4934 & 7484 & 11219 & 28068 & 41450 & 149948 \\
\hline 25 & & MMV & 6.43847 & 1.10027 & 0.08350 & 304.9 & - & 2562 & 4315 & 6046 & 8191 & 15158 & 19208 & 38805 \\
\hline 25 & & OPN & 6.38270 & 1.22503 & 0.00709 & 165.5 & (5) 45 & 2841 & 5066 & 7362 & 10307 & 20369 & 26467 & 57562 \\
\hline 25 & PAG & MOL & 653.192 & 398.602 & -0.51895 & 453.1 & - & 2287 & 3882 & 5673 & 8223 & 19187 & 27557 & 91326 \\
\hline 25 & & OPN & 720.342 & 460.704 & -0.57561 & 212.5 & (10) 84 & 2755 & 4906 & 7439 & 11192 & 28519 & 42565 & 160487 \\
\hline 26 & & MOL & 169.825 & 39.034 & 0.03898 & 7.9 & - & 254 & 287 & 311 & 334 & 385 & 406 & 472 \\
\hline 26 & & OPN & 172.115 & 40.304 & 0.02645 & 7.7 & (12) 131 & 260 & 296 & 322 & 347 & 403 & 427 & 502 \\
\hline 26 & & MMV & 5.22410 & 0.24629 & 0.13407 & 7.6 & - & 255 & 286 & 309 & 331 & 380 & 401 & 470 \\
\hline 26 & & OPN & 5.22655 & 0.25159 & 0.05087 & 7.2 & (11) 114 & 257 & 290 & 314 & 338 & 390 & 413 & 488 \\
\hline 26 & LOG & MOL & 185.141 & 25.224 & -0.14524 & 7.2 & - & 250 & 287 & 317 & 350 & 440 & 485 & 673 \\
\hline 26 & & OPN & 185.141 & 27.746 & -0.14524 & 6.5 & (1) 9 & 257 & 297 & 330 & 367 & 465 & 515 & 722 \\
\hline 27 & & MOL & 264.123 & 93.736 & -0.03176 & 16.3 & - & 483 & 580 & 654 & 728 & 908 & 988 & 1267 \\
\hline 27 & & OPN & 260.254 & 92.497 & -0.09044 & 13.5 & (20) 184 & 491 & 603 & 693 & 788 & 1032 & 1148 & 1590 \\
\hline 27 & & MMV & 5.69544 & 0.38280 & 0.04381 & 15.8 & - & 486 & 582 & 654 & 727 & 899 & 976 & 1244 \\
\hline 27 & & OPN & 5.70070 & 0.38211 & 0.04334 & 14.0 & (11) 108 & 489 & 587 & 662 & 737 & 917 & 998 & 1285 \\
\hline 27 & GVE & MOL & 298.941 & 63.007 & -0.19039 & 15.1 & - & 471 & 574 & 662 & 762 & 1048 & 1201 & 1879 \\
\hline 27 & & OPN & 298.941 & 69.308 & -0.19039 & 12.9 & (1) 9 & 488 & 602 & 699 & 808 & 1123 & 1291 & 2037 \\
\hline 28 & & SEX & 271.602 & 93.018 & 0.00843 & 14.1 & - & 479 & 565 & 629 & 691 & 835 & 896 & 1096 \\
\hline 28 & & OPN & 268.694 & 94.484 & -0.03379 & 11.8 & (7) 71 & 490 & 588 & 663 & 739 & 922 & 1004 & 1290 \\
\hline 28 & & MMV & 5.71768 & 0.36916 & 0.11273 & 12.4 & - & 488 & 582 & 652 & 722 & 888 & 962 & 1220 \\
\hline 28 & & OPN & 5.72233 & 0.36126 & 0.02357 & 12.3 & (4) 61 & 486 & 577 & 645 & 713 & 874 & 946 & 1194 \\
\hline 28 & LOG & MOL & 308.338 & 61.857 & -0.15437 & 11.3 & - & 470 & 562 & 638 & 722 & 953 & 1071 & 1568 \\
\hline 28 & & OPN & 308.338 & 68.042 & -0.15437 & 10.9 & (1) 9 & 486 & 588 & 671 & 764 & 1018 & 1148 & 1694 \\
\hline 29 & & MOL & 21.178 & 2.704 & 0.57722 & 0.333 & - & 24.6 & 25.1 & 25.4 & 25.5 & 25.7 & 25.8 & 25.8 \\
\hline 29 & & OPN & 21.157 & 2.935 & 0.66494 & 0.252 & (4) 46 & 24.6 & 25.0 & 25.2 & 25.4 & 25.5 & 25.5 & 25.6 \\
\hline 29 & & MML & 3.07058 & 0.11604 & -1.20543 & 0.420 & - & 24.4 & 25.0 & 25.3 & 25.5 & 25.9 & 25.9 & 26.1 \\
\hline 29 & & OPN & 3.06430 & 0.13545 & -0.23489 & 0.260 & (3) 38 & 25.4 & 26.9 & 27.8 & 28.7 & 30.5 & 31.1 & 33.2 \\
\hline 29 & GVE & MOL & 22.031 & 1.324 & 0.15284 & 0.444 & - & 24.5 & 25.4 & 25.9 & 26.4 & 27.3 & 27.7 & 28.6 \\
\hline 29 & & OPN & 22.026 & 1.467 & 0.19819 & 0.293 & (3) 40 & 24.6 & 25.5 & 26.0 & 26.5 & 27.3 & 27.5 & 28.2 \\
\hline 30 & & MOL & 41.793 & 11.095 & 0.07426 & 1.5 & - & 65 & 73 & 79 & 85 & 97 & 102 & 116 \\
\hline 30 & & OPN & 41.445 & 11.363 & 0.03287 & 1.3 & (10) 98 & 66 & 76 & 83 & 90 & 105 & 112 & 132 \\
\hline 30 & & MMV & 3.82453 & 0.27009 & 0.14311 & 1.4 & - & 65 & 74 & 80 & 86 & 101 & 107 & 127 \\
\hline 30 & & OPN & 3.82584 & 0.27985 & 0.02164 & 1.3 & (5) 67 & 66 & 75 & 82 & 88 & 104 & 110 & 132 \\
\hline 30 & GVE & MOL & 46.034 & 7.031 & -0.12331 & 1.4 & - & 64 & 73 & 81 & 89 & 112 & 123 & 167 \\
\hline 30 & & OPN & 45.908 & 7.343 & -0.15104 & 1.0 & (6) 62 & 65 & 76 & 85 & 95 & 122 & 135 & 193 \\
\hline 31 & & MOL & 58.959 & 13.476 & 0.04642 & 1.6 & - & 88 & 99 & 107 & 115 & 132 & 139 & 160 \\
\hline 31 & & OPN & 59.519 & 12.980 & 0.02113 & 1.7 & (3) 29 & 88 & 100 & 108 & 116 & 135 & 143 & 168 \\
\hline 31 & & MML & 4.16632 & 0.23395 & 0.19732 & 1.7 & - & 87 & 99 & 107 & 115 & 134 & 142 & 170 \\
\hline 31 & & OPN & 4.16678 & 0.24590 & 0.01540 & 1.5 & (3) 61 & 88 & 99 & 107 & 115 & 132 & 139 & 162 \\
\hline 31 & PAG & MOL & 64.207 & 8.672 & -0.14059 & 2.3 & - & 87 & 99 & 109 & 120 & 150 & 165 & 228 \\
\hline 31 & & OPN & 64.609 & 8.889 & -0.14059 & 2.2 & (2) 14 & 88 & 100 & 111 & 122 & 153 & 168 & 232 \\
\hline
\end{tabular}




\section{Simbología:}

NR número de registro según cuadro 1.

DA distribución adecuada según figura 2.

PT3 Pearson tipo III.

PAG Pareto generalizada.

LOG logística generalizada.

GVE general de valores extremos.

LN3 Log-Normal de 3 parámetros.

MA método de ajuste:

MOL método de momentos $L$.

OPN método de optimización numérica.

MMV método de máxima verosimilitud.

SEX método de sextiles.

MML método de momentos en el dominio logarítmico.

MMM método de mezcla de momentos.

MME método de máxima entropía.

MPD método de promedios diversos.

u parámetro de ubicación.

$\alpha \quad$ parámetro de escala.

$k$ parámetro de forma.

Ym media logarítmica.

Sy desviación estándar logarítmica.

$g_{c} \quad$ coef. de asimetría logarítmico corregido.

EEA error estándar de ajuste $\left(\mathrm{m}^{3} / \mathrm{s}, \mathrm{mm}, \mathrm{km} / \mathrm{h}\right)$.

\section{Referencias}

Campos-Aranda D.F. Contraste de cinco métodos de ajuste de la distribución GVE en 31 registros históricos de eventos máximos anuales. Ingeniería Hidráulica en México, volumen XVI (número 2), abril-junio de 2001: 77-92.

Campos-Aranda D.F. Contraste de seis métodos de ajuste de la distribución Log-Pearson tipo III en 31 registros históricos de eventos máximos anuales. Ingeniería Hidráulica en México, volumen XVII (número 2), abril-junio de 2002a: 77-97.

Campos-Aranda D.F. Ajuste de la distribución Log-Pearson tipo III por medio de optimización numérica no restringida. Ingeniería Hidráulica en México, volumen XVII (número 4), octubrenoviembre de 2002b: 115-128.

Campos-Aranda D.F. Introducción a los Métodos Numéricos: Software en Basic y aplicaciones en Hidrología Superficial, capítulo 9:
Optimización numérica, pp. 172-211, San Luis Potosí, SLP, Librería Universitaria Potosina, 2003, 222 p.

Greenwood J.A., Landwehr J.M., Matalas N.C., Wallis J.R. Probability Weighted Moments: Definition and Relation to Parameters of Several Distributions Expressible in Inverse Form. Water Resources Research, volumen 15, 1979: 1049-1054.

Hosking J.R.M., Wallis J.R. Regional Frequency Analysis. An Approach Based on L-Moments, Appendix: L-Moments for Some Specific Distributions, pp. 191-209, Cambridge, Inglaterra, Cambridge University Press, 1997, 224 p.

Kuester J.L., Mize J.H.. Optimization Techniques with Fortran, ROSENB Algorithm, pp. 320-330, Nueva York, USA, McGrawHill Book Co., 1973.

Maidment D.R. Hydrology, en: Handbook of Hydrology, pp. 1.11.15, editor Maidment D.R. , Nueva York, USA, McGraw-Hill, Inc., 1993.

Mansell M.G. Rural and Urban Hydrology, capítulo 8: The Analysis and Predictions of Flows, pp. 319-354, Londres, Inglaterra, Thomas Telford Publishing Ltd., 2003, 411 p.

Natural Environment Research Council (NERC). Flood Studies Report, volumen I: Hydrological Studies, Londres, Inglaterra, $1975,550 \mathrm{p}$.

Ponce V.M. Engineering Hydrology. Principles and Practices., capítulo 6: Frequency Analysis, pp. 205-232, Englewood Cliffs, NJ, USA, Prentice Hall, 1989, 640 p.

Shaw E.M., Beven K.J., Chappel N.A., Lamb R. Hydrology in Practice, capítulo 13: Estimating Floods and Low Flows in the UK, pp. 322-350, 4a ed., Londres, Inglaterra, Spon Press, 2011, 543 p.

Water Resources Council (WRC). Guidelines for Determining Flood Flow Frequency. Bulletin \# 17A of the Hydrology Committee, Washington DC, USA, 1977.

\section{Este artículo se cita:}

Citación Chicago

Campos-Aranda, Daniel Francisco. Contraste de la distribución logística generalizada en 31 registros históricos de eventos máximos anuales. Ingeniería Investigación y Tecnología XIV, 01 (2013): 113-123.

\section{Citación ISO 690}

Campos-Aranda D.F. Contraste de la distribución logística generalizada en 31 registros históricos de eventos máximos anuales. Ingeniería Investigación y Tecnología, volumen XIV (número 1), enero-marzo 2013: 113-123. 


\section{Semblanza del autor}

Daniel Francisco Campos-Aranda. Obtuvo el título de ingeniero civil en diciembre de 1972, en la entonces Escuela de Ingeniería de la UASLP. Durante el primer semestre de 1977, realizó en Madrid, España un diplomado en hidrología general y aplicada. Posteriormente, durante 1980-1981 llevó a cabo estudios de maestría en ingeniería en la especialidad de hidráulica, en la División de Estudios de Posgrado de la Facultad de Ingeniería de la UNAM. En esa misma institución, inició (1984) y concluyó (1987) el doctorado en ingeniería con especialidad en aprovechamientos hidráulicos. Ha publicado artículos, principalmente en revistas mexicanas de excelencia: 40 en Tecnología y Ciencias del Agua (antes Ingeniería Hidráulica en México), 14 en Agrociencia y 11 en Ingeniería. Investigación y Tecnología. Fue investigador nacional (nivel I) desde el $1^{\circ}$ de julio de 1991 hasta el 31 de diciembre del 2007. Actualmente es profesor jubilado de la UASLP, desde el $1^{\circ}$. de febrero del 2003. En noviembre de 1989 obtuvo la medalla Gabino Barreda de la UNAM y en 2008 le fue otorgado el Premio Nacional "Francisco Torres H." de la AMH, a la práctica profesional de la hidráulica. 